\title{
Evaluación de calidad del agua en la Quebrada Jui, afluente del río Sinú, Colombia
}

\section{Water quality assessment in Quebrada Jui, tributary of the Sinú river, Colombia}

\author{
Urledys Hernández-Álvarez ; José Pinedo-Hernández²; Roberth Paternina-Uribe ${ }^{3}$; José Luis Marrugo-Negrete ${ }^{4}$
}

\begin{abstract}
${ }^{1}$ Lic en Ciencias Naturales y Educación Ambiental, M.Sc. Universidad de Córdoba, Maestría en Ciencias Ambientales. Montería - Córdoba, Colombia e-mail: uyhernandezalvarez@correo.unicordoba.edu.co; (D) https://orcid.org/0000-0001-5964-8344

²Químico, M.Sc. Universidad de Córdoba, Maestría en Ciencias Ambientales. Montería - Córdoba, Colombia; e-mail: jjoaquinpinedo@correo.unicordoba.edu.co; (D) https://orcid.org/0000-0002-4288-1788

${ }^{3}$ Químico Farmacéutico, M.Sc. Universidad de Córdoba, Maestría en Ciencias Ambientales. Montería - Córdoba, Colombia; e-mail: rpaternina@correo. unicordoba.edu.co; (D) https://orcid.org/0000-0002-1994-0225
\end{abstract}

${ }^{4}$ Ing. Químico, Ph.D. Universidad de Córdoba, Grupo de Aguas, Química Aplicada y Ambiental, Laboratorio de Toxicología y Gestión Ambiental. Montería Córdoba, Colombia; e-mail: jmarrugo@correo.unicordoba.edu.co; (D) https://orcid.org/0000-0002-3181-7529

Cómo citar: Hernández-Álvarez, U.; Pinedo-Hernández, J.; Paternina-Uribe, R.; Marrugo-Negrete, J.L. 2021. Evaluación de calidad del agua en la Quebrada Jui, afluente del río Sinú, Colombia. Rev. U.D.C.A Act. \& Div. Cient. 24(1):e1678. http://doi.org/10.31910/rudca. v24.n1.2021.1678

Artículo de acceso abierto publicado por Revista U.D.C.A Actualidad \& Divulgación Científica, bajo una licencia Creative Commons CC BY-NC 4.0

Publicación oficial de la Universidad de Ciencias Aplicadas y Ambientales U.D.C.A, Institución de Educación Superior Acreditada de Alta Calidad por el Ministerio de Educación Nacional.

Recibido: agosto 30 de $2020 \quad$ Aceptado: febrero 11 de $2021 \quad$ Editado por: Ingeborg Zenner de Polanía

\section{RESUMEN}

La microcuenca de la quebrada Jui, enmarcada dentro de la cuenca alta del río Sinú, región Caribe de Colombia, es uno de los recursos hídricos utilizados por la población rural para consumo, riego y saneamiento; sin embargo, recibe cargas contaminadas de centros poblados en su área de influencia, que corresponden al vertimiento de aguas residuales, afluentes de laguna de oxidación, actividades ganaderas, extracción de arena, agricultura de pancoger y cultivos no formales. El objetivo de este estudio fue determinar el índice de calidad del agua (ICA) y establecer la relación entre los parámetros fisicoquímicos y microbiológicos con las diferentes fuentes antropogénicas, para establecer origen común de contaminación. Además, el análisis de plaguicidas organoclorados y organofosforados, para conocer la influencia de las actividades agrícolas. Se tomaron muestras en seis estaciones, en el periodo seco y húmedo del 2018. Las concentraciones de los parámetros fisicoquímicos y microbiológicos, se analizaron empleando los métodos estandarizados de la American Public Health Association. Los plaguicidas, se cuantificaron por el método cromatografía de gases con espectrometría de masas (GC-MS). En promedio, el ICA fue 74,1, clasificando la fuente hídrica con buena calidad, exceptuando las estaciones (E4 y E5), en el periodo húmedo, cuya clasificación fue media, debido al aporte de coliformes fecales y turbidez. La concentración de plaguicidas, se presentó por debajo del límite de detección del método. En general, el análisis estadístico multivariado indica que las fuentes de contaminación derivan de aguas residuales domésticas, escorrentía agrícola, excretas del ganado porcino y minería de arena. 
Palabras clave: Índice de calidad del agua; Río Sinú; Recursos hídricos; Origen contaminación.

\section{ABSTRACT}

The micro-base of the Jui creek, framed within the upper Sinú River basin, Colombia Caribbean region, is one of the water resources used by the rural population for consumption, irrigation and sanitation. However, it receives contaminated loads from urban centers in its area of influence that correspond to the discharge wastewater, oxidation lagoon tributaries, livestock activities, sand extraction, agriculture and non-formal crops. The objective of this study was to determine the water quality index (ICA) and establish the relationship between the physical-chemical and microbiological parameters with the different anthropogenic sources to establish a common source of contamination. In addition, the analysis of organochlorinated and organophosphate pesticides to understand the influence of agricultural activities. Samples were taken in six seasons, in the dry and humid period of 2018. Concentrations of physical-chemical and microbiological parameters were analyzed using the American Public Health Association's standardized methods. The pesticides were quantified by the gas chromatography method with mass spectrometry (GC-MS). On average the WQI was 74.1 classifying the water source with good quality, except the seasons (E4 and E5) in the wet period, the classification of which was average, due to the contribution of fecal coliforms and turbidity. The concentration of pesticides was below the detection limit of the method. In general, multivariate statistical analysis indicates that sources of pollution are from domestic wastewater, agricultural runoff, pig excretions and sand mining.

Keywords: Water quality index; Sinu river; Water resources; Contamination origin.

\section{INTRODUCCIÓN}

Colombia, se ubica entre los países con mayor riqueza hídrica en el mundo (IDEAM, 2018); no obstante, considerando el impacto antrópico sobre diferentes cuerpos de agua, su disponibilidad es mucho menor, al superar su capacidad de autodepuración, lo cual, conlleva a obtener un índice de calidad bajo, que indica deterioro en la calidad del agua, donde la agrupación simplificada de algunos constituyentes representan un componente en el manejo global integrado de los recursos hídricos (Gualdrón Durán, 2016; Hoyos Chaverra et al. 2018; Pradana Pérez et al. 2019; Tosic et al. 2019).

Registros obtenidos por el Ministerio de Ambiente, Vivienda y Desarrollo Territorial, en el 2004, demuestran la problemática de contaminación, a nivel nacional, con, aproximadamente, 1.300 cuerpos de agua contaminados, destacándose, en el interior del país: los ríos Bogotá, Medellín, Cali y Sogamoso, donde la contaminación de las diferentes fuentes hídricas relacionadas con la calidad de parámetros fisicoquímicos llegó a generar condiciones anóxicas. Esta situación, asociada a que más del $80 \%$ de los asentamientos urbanos se abastecen de fuentes naturales, como ríos, arroyos, quebradas, entre otros, generan riesgo potencial en poblaciones, debido a su utilización en diferentes actividades domésticas, ocasionando que la comunidad, en general, sea altamente vulnerable (MAVDT, 2010). En el Caribe colombiano, el río Sinú, principal arteria fluvial del departamento de Córdoba, tiene diferentes microcuencas, que aportan y que pueden generar contaminación (Lans et al. 2008; Feria et al. 2010; Marrugo-Negrete et al. 2017), entre ella, la quebrada Jui, ubicada en la cuenca alta de esta vertiente fluvial y una de las fuentes principales de abastecimiento, para el desarrollo de diferentes actividades en poblaciones aledañas, en el municipio de Tierralta; receptor de residuos sólidos, aguas residuales domésticas, afluentes de la laguna de oxidación, escorrentías agrícolas y ganaderas. Además, con actividades de minería de arena y deforestación en la ribera, con zonas boscosas reemplazadas, en gran proporción, por cultivos no formales y agrícolas, donde la aplicación de plaguicidas organoclorados y organofosforados, en el pasado, era de uso común, por su efectividad en el control de plagas (CVS, 2006). Cabe resaltar, que la aplicación de plaguicidas organoclorados en Colombia fue prohibida desde la década de los años setenta, acorde a la Resolución 447 de 1974 del Ministerio de Agricultura; sin embargo, la persistencia de los residuos y metabolitos generan problemas de contaminación en diferentes matrices ambientales (Svingen et al. 2018; Sharma et al. 2020); como es el caso reportado en la ciénaga grande del bajo Sinú, donde se encontraron concentraciones de plaguicidas, como $\alpha-\mathrm{BCH}, \beta-\mathrm{BCH}, \boldsymbol{\gamma}-\mathrm{BCH}$, aldrin y heptacloro epóxido (Lans et al. 2008). Además, Marrugo-Negrete et al. (2014) en suelos agrícolas en la cuenca media y baja del río Sinú, encontraron $\beta$-BCH, lindano, $\alpha$-clordano, $\beta$ - endosulfan, 4,4'-DDT y 4,4'-DDE. Racero Casarrubia et al. (2021) reportaron concentraciones de $\alpha$ -BCH, $\beta$-BCH, $\boldsymbol{\gamma}$-BCH, endrin, pp-DDE, pp-DDT, en murciélagos en el sector Manso-Tigre en Tierralta, Córdoba. Lo anterior, refleja la problemática ambiental por este tipo de contaminantes en zonas de influencia al área del estudio.

Aunque es conocido que existen diferentes actividades antropogénicas que pueden llegar a generar problemas de contaminación, no se encuentran evaluaciones de la calidad del agua en la quebrada Jui, recurso de gran importancia para diez comunidades aledañas con, aproximadamente, 3.535 habitantes, según último censo (Sisben, 2009). Las necesidades del agua son el consumo, la preparación de alimentos y el saneamiento personal, razón por la cual, este estudio presenta el índice de calidad del agua (ICA) y la relación de los parámetros fisicoquímicos y microbiológicos en época seca y húmeda, con las diferentes fuentes antropogénicas, para establecer origen común de contaminación; además, el análisis de plaguicidas organoclorados y organofosforados, para conocer la influencia de las actividades agrícolas, estableciendo un índice de calidad del agua, como referente para evaluar la vulnerabilidad del recurso hídrico.

\section{MATERIALES Y MÉTODOS}

El siguiente trabajo, se realizó bajo la modalidad investigativa de tipo descriptiva correlacional de campo, con el fin de evaluar la calidad del agua en la microcuenca quebrada Jui, en el municipio de Tierralta, departamento de Córdoba, mediante la determinación del índice de calidad del agua, plaguicidas e identificación de las principales fuentes de contaminación. 
Área de estudio. La microcuenca de la quebrada Jui, se encuentra ubicada en la cuenca alta del río Sinú, al sur oriente del casco urbano del municipio de Tierralta, departamento de Córdoba (Figura 1). Nace en el cerro Murrucucú, jurisdicción del parque Natural Paramillo, a una altitud aproximada de $1.386 \mathrm{~m}$ s.n.m. Tiene una extensión aproximada de $290,4 \mathrm{~km}^{2}$ y un recorrido de $36,9 \mathrm{~km}$, de sur a norte, hasta desembocar en el río Sinú, con una altitud de $34 \mathrm{~m}$ s.n.m. Recibe cargas contaminadas de centros poblados en su área de influencia, como el vertimiento de aguas residuales, afluentes de laguna de oxidación, actividades ganaderas, extracción de arena, agricultura de pancoger y otros cultivos no formales. Además, tiene la característica de ser utilizada por la población rural para consumo, riego y saneamiento (CVS, 2006).
Recolección y análisis de muestras. La figura 1 muestra las estaciones de muestreo seleccionadas en la microcuenca quebrada Jui (E1 a E6), a través de campañas de muestreo realizadas en marzo (época seca) y junio (época húmeda) 2018. Las estaciones de muestreo fueron seleccionadas en función de la dinámica flujo de la microcuenca, facilidad de acceso, seguridad, flujo permanente de agua, influencia de actividades antrópicas y ubicación de zonas de perímetro urbano (E3, E4, E5) y suburbanas o rurales (E1, E2, E6).

Las muestras fueron tomadas en recipientes de vidrio ámbar, previamente esterilizados, de 1L, para los parámetros fisicoquímicos y $0,25 \mathrm{~L}$, para las variables microbiológicas, refrigeradas a $4^{\circ} \mathrm{C}$ y transportadas al laboratorio en neveras plásticas, con monitoreo

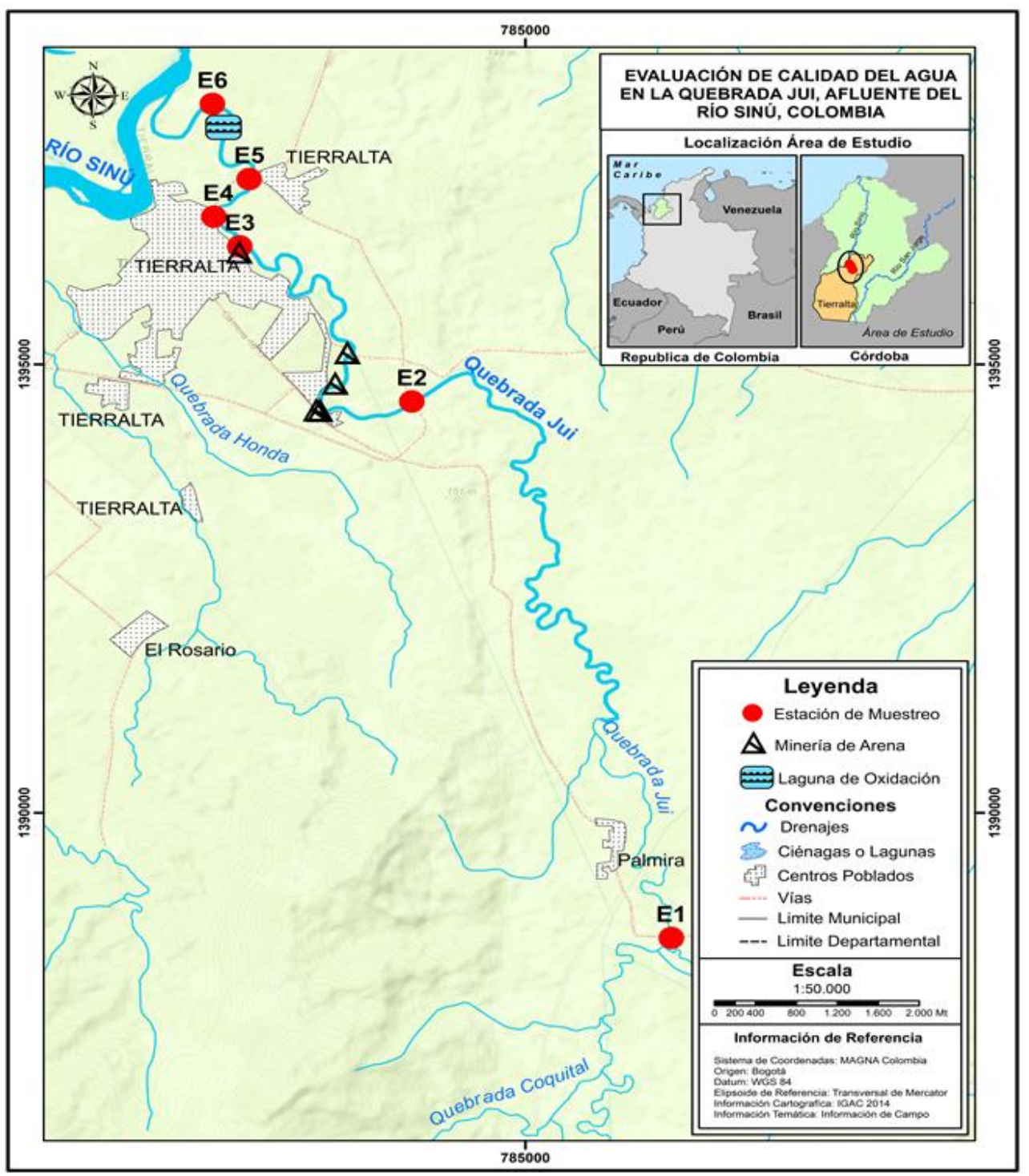

Figura 1. Microcuenca de la quebrada Jui, municipio de Tierralta, departamento Córdoba. E1: Puente de Palmira vía Revuelto; E2: Puente antes del casco urbano de Tierralta; E3: Barrio 20 de Julio antes del hospital; E4: Barrio el prado después del hospital; E5: Puente de acceso a Tierralta; E6: Desembocadura de la quebrada Jui. Fuente cartográfica: IGAC, 2014. Año 2020; Software ArcGIS, extensión ArcMAP 10.5. 
de temperatura, para control de cadena de frío. Posteriormente, caracterización físico-química y microbiológica, mediante análisis de temperatura (T), turbidez (Turb), sólidos totales (ST), $\mathrm{pH}$, oxígeno disuelto (OD), demanda química de oxígeno (DQO), demanda bioquímica de oxígeno ( $\mathrm{DBO}_{5}$ ), nitratos $\left(\mathrm{NO}_{3}\right)$, fosfatos $\left(\mathrm{PO}_{4}{ }^{3}\right)$, coliformes totales (CT) y fecales (CF), acorde con métodos normalizados por APHA (2017); además, se determinó la concentración de plaguicidas organoclorados y organofosforados por métodos cromatográficos. Las muestras de agua, se sometieron a técnicas de extracción fase sólida y líquido-líquido con diclorometano y acetato de etilo. Los extractos orgánicos fueron mezclados y rotaevaporados. La determinación de los plaguicidas cipermetrina y clorpirifos, se realizó por cromatografía líquida de alta eficiencia, con detector de arreglo de diodos (HPLC-DAD) y, el resto de los plaguicidas, por cromatografía gaseosa, con detector de espectrometría de masas (GC-MS). Se compararon los tiempos de retención y se calcularon las áreas bajo la curva de muestras y estándares según patrones analizados (Ortiz-Romero et al. 2015). La cuantificación de los plaguicidas organoclorados, se realizó empleando estándar interno: clorfenson pestanal ${ }^{\circledR}$, Sigma aldrich. El límite de detección para los 11 compuestos: $\alpha$-BHC, $\beta$-BHC, Ү-BHC, $\delta$-BHC, heptacloro, aldrín, heptacloro-epoxido, endosulfan, p-p' - DDE, dieldrín, endrin, endosulfan II, m-p'DDD, endrin-aldehido, endosulfan sulfato, p-p'DDT, endrin-eetona, estuvo entre 0,05 y $0,25 \mathrm{ug} / \mathrm{L}$. Los $\% \mathrm{R}$ para los analitos evaluados en muestras fortificadas estuvieron entre el 80 y 120\%. Para el caso de los plaguicidas organofosforados, como diclorfos, Mocap, malathion, methilparation, Romel, clorpirifos y disulfoton, fueron cuantificados mediante estándar interno: organophosphorous pest mix Supelco. El límite de detección estuvo en el rango de 3 a 5ug/L y los $\%$ R estuvieron por encima del $70 \%$.

Índice de calidad del agua (ICA). El cálculo del ICA permite realizar un análisis general de la calidad del agua en diferentes niveles y determinar la vulnerabilidad del cuerpo frente a amenazas potenciales (Soni \& Thomas, 2014). Para la determinación del ICA, se empleó la ecuación de promedio aritmético ponderado, acorde con lo descrito por Sutadian et al. (2016):

$$
I C A=\sum_{i=1}^{9} S u b_{i} \cdot w_{i}
$$

Donde: Subi. Sudíndice del parámetro i; $W_{i}$. Factor de ponderación para el Sudíndice i. Los valores de calidad se calcularon a partir de nueve parámetros establecidos por la National Sanitation Foundation - NSF de Estados Unidos (Fontalvo Julio \& TamarisTurizo, 2018), con factores de ponderación, dentro de la puntuación total del índice: OD, 0,17 ; $\mathrm{CF}, 0,15 ; \mathrm{pH}, 0,12 ; \Delta \mathrm{T}, 0,10 ; \mathrm{DBO}_{5}$, 0,10; $\mathrm{NO}_{3}, 0,10 ; \mathrm{PO}_{4}{ }^{3-}, 0,10$; Turb, 0,08; ST, 0,08. Los resultados, se interpretaron en función de la categorización: Nivel 1 (calidad excelente 91-100); Nivel 11 (calidad buena 71-90); Nivel 111 (calidad media 51-70); Nivel IV (calidad mala 26-50); Nivel V (calidad muy mala 0-25) (Gil-Marín et al. 2018; Pradana Pérez et al. 2019).
Análisis estadístico. Los resultados para cada muestra, se calcularon como la media de las determinaciones por triplicado. Se utilizó la prueba Tukey, para evaluar si había diferencias significativas entre las concentraciones promedio de los parámetros fisicoquímicos y microbiológicos entre periodos de muestreo. Para identificar relaciones significativas y origen común entre parámetros, se realizó un análisis de Pearson, componentes principales (ACP) y conglomerados. El análisis estadístico, se efectuó con SPSS v23.0.0.0, utilizando, como criterio de significancia, $p<0,05$.

\section{RESULTADOS Y DISCUSIÓN}

La tabla 1 muestra la concentración promedio de parámetros fisicoquímicos y microbiológicos por estaciones y periodos de muestreo. En general, para las diferentes estaciones no se presenta variabilidad espacial y diferencias estadísticamente significativas ( $>00,05)$; no obstante, al comparar las estaciones de perímetro urbano (E3, E4, E5) y suburbanas (E1, E2, E6), se observa que los parámetros presentaron mayores concentraciones en las estaciones ubicadas en el área de predominio de la zona urbana, caracterizadas por la influencia de actividades antrópicas, como son vertimiento de aguas residuales domésticas, residuos sólidos, ganadería porcina y minería de arena.

En cuanto a los periodos de muestreo, las concentraciones promedio de los parámetros en el periodo húmedo, fueron mayores con respecto a las obtenidas en la época seca, excepto la $\mathrm{DQO}$ y el $\mathrm{NO}_{3}$, que no mostraron diferencia estadísticamente significativa entre los periodos de muestreo. La temperatura, $\mathrm{pH}$ y el OD, se encuentran dentro del rango óptimo para el desarrollo de la biota acuática (MADS, 2015; Gualdrón Durán, 2016). Independientemente del periodo de muestreo, los resultados de $\mathrm{DQO}>5 \mathrm{mgO}_{2} / \mathrm{L}$ indican contaminación en el recurso hídrico (Restrepo Mejía \& Tobón Mejía, 2002);sin embargo, las variaciones del parámetro $\mathrm{DBO}_{5}$ vs DQO entre periodos de muestreo son características de baja contaminación orgánica, indicando la presencia de sustancias inorgánicas, que incrementan los resultados de DQO en época seca, mientras que, en época húmeda, el aporte de condiciones anóxicas o cargas de material orgánico pueden causar incrementos en estos parámetros (Torres et al. 2010); no obstante, la rápida capacidad de oxigenación y asimilación de la materia orgánica por la corriente del recurso hídrico en el periodo húmedo, se refleja en los valores de $\mathrm{OD}(>5,0 \mathrm{mg} / \mathrm{L})$ y parámetros, como $\mathrm{NO}^{3-}$ y $\mathrm{PO}_{4}{ }^{3-}$, que presentaron valores $<0,5 \mathrm{mg} / \mathrm{L}$, indicando que el recurso hídrico no demuestra procesos de eutrofización, independientemente de las diferentes fuentes antropogénicas de contaminación (Antonio Paiba \& Monroy Ávila, 2015). A diferencia, los coliformes totales, fecales y la turbidez, constituyen un problema de contaminación del recurso, al superar los límites permisibles para actividades de riego y consumo (MPS-MAVDT, 2007; MADS, 2015; Morales-Durán et al. 2018). Lo anterior representa riesgo potencial en la salud humana y ambiental por la utilización del recurso, con mayor impacto en el periodo húmedo, donde las concentraciones de coliformes totales y fecales superan, en promedio, ocho veces el valor obtenido en época seca y la turbidez, en promedio, supera en una relación de 96 veces, relacionado con las concentraciones de ST, en época 
Tabla 1. Concentración promedio de parámetros fisicoquímicos y microbiológicos en función de estaciones y periodos de muestreo en la microcuenca quebrada Jui.

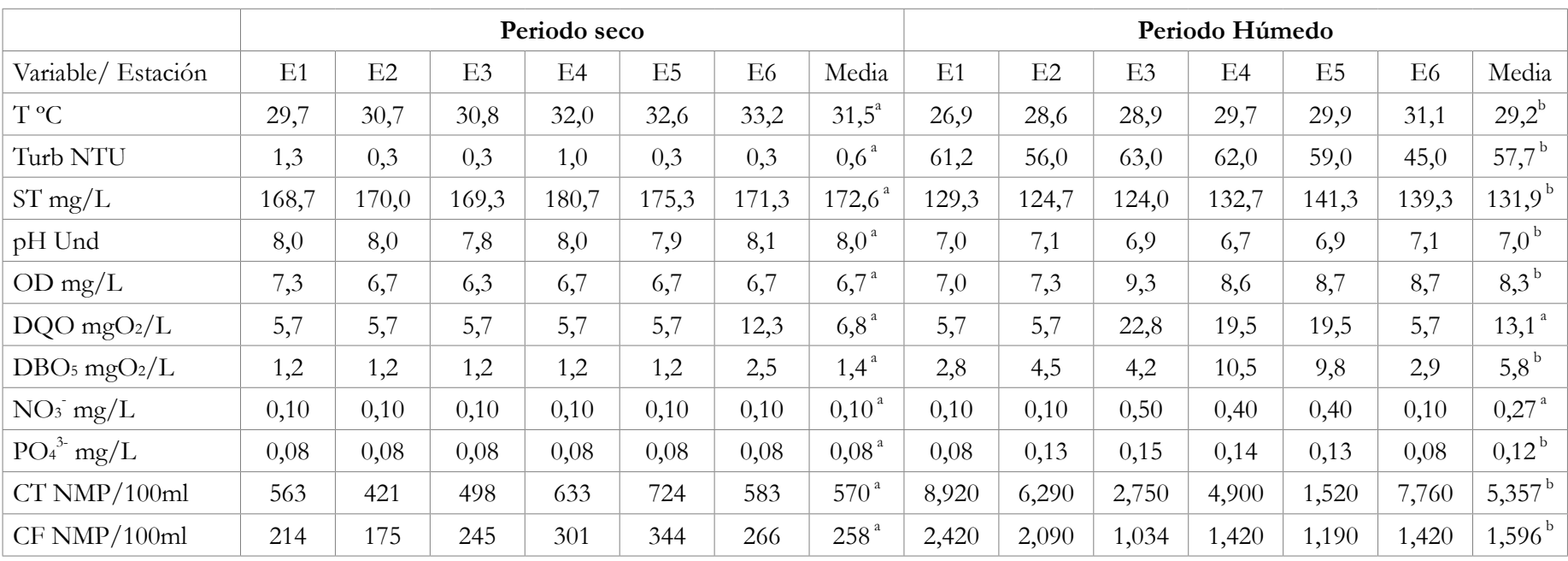

Medias con diferentes letras indican diferencia significativa entre periodos de muestreo $(p<0,05)$.

húmeda, extracción de arena y erosión, en el caso de la turbidez; actividades ganaderas, agrícolas y vertimiento de aguas residuales, para coliformes totales y fecales (Mora \& Calvo, 2010; Gualdrón Durán, 2016; Martínez Santos, 2018; González-Dávila et al. 2019).

Cabe resaltar, que la zona de estudio presenta actividades agrícolas que pueden generar contaminación, por la aplicación de plaguicidas que, al presente, están prohibidos, asociados desde el manejo inadecuado de los mismos, sus empaques, tipos de plaguicidas y aplicación, los cuales, por escorrentía, pueden llegar a ecosistemas acuáticos (CVS, 2006); sin embargo, los plaguicidas analizados presentaron resultados por debajo del límite de detección del método, debido a su rápida absorción por sedimentos, fenómeno de dilución, solubilidad y descomposición (Rice et al. 2002; Usma Rios et al. 2008). Además, adsorción de partículas en superficie del suelo, volatilización, hidrólisis, condiciones físico-químicas del medio, $\mathrm{pH}$, materia orgánica, potencial redox, humedad y estructura molecular, que influyen en la retención de este tipo de contaminantes en otras matrices ambientales, impidiendo su ingreso a ecosistemas acuáticos (Cavanagh et al. 1999; Sweetman et al. 2005; Lewis et al. 2016). A pesar de no encontrar en este estudio niveles de plaguicidas, existen reportes de contaminación que reflejan la persistencia de estos contaminantes y procesos de bioacumulación; por ejemplo, el estudio realizado por Racero Casarrubia et al. (2021) reportaron contaminación por plaguicidas organoclorados en mamíferos en zonas de influencia al área de estudio; Marrugo-Negrete et al. (2014) indicaron concentración de estos contaminantes en suelos agrícolas en la cuenca media y baja del río Sinú, demostrando que, a pesar de ser prohibidos, aún se presentan en suelos agrícolas y debido a su persistencia son bioacumulados y biomagnificados en la red trófica.

En general, los resultados de plaguicidas son similares $(<\mathrm{LDM})$ a los reportados, a nivel nacional, en el río Otún, Risaralda (Usma Rios et al. 2008) y la parte baja del río Córdoba, Magdalena (Fontalvo
Julio \& Tamaris-Turizo, 2018) e inferiores a los reportados por Lans et al. (2008), en la cuenca del río Sinú, donde se detectaron concentraciones de $\boldsymbol{\alpha}$-BCH $(0,112 \mu \mathrm{g} / \mathrm{L}), \boldsymbol{\beta}-\mathrm{BCH}(0,5861 \mu \mathrm{g} / \mathrm{L})$, $\gamma$-BCH $(0,115 \mu \mathrm{g} / \mathrm{L})$, Aldrín $(0,280 \mu \mathrm{g} / \mathrm{L})$ y Heptacloro-epóxido $(0,549 \mu \mathrm{g} / \mathrm{L})$, por encima del límite admisible de $0,1 \mu \mathrm{g} / \mathrm{L}$, acorde con la normatividad colombiana para agua potable, según Decreto 1076 de 2015 y Resolución 2115 de 2007 (MPS-MAVDT, 2007; MADS, 2015).

Índice de calidad de aguas (ICA). La figura 3 muestra la clasificación de la calidad del agua de la quebrada Jui. Los resultados del ICA, se presentaron entre 66,7 - 79,0 (promedio: 74,1), indicando que la calidad del agua es de nivel II (aguas de buena calidad). En el periodo seco, todas las estaciones mostraron calidad de agua 11 y en el periodo húmedo, todas las estaciones, con excepción de E4 y E5, también fueron de buena calidad; la disminución en la calidad de estas dos estaciones, se debe al aporte de coliformes fecales y turbidez. En general, las estaciones que se encuentran fuera del perímetro urbano (E1, E2, E6) presentan calidad buena, a diferencia de las estaciones cercanas a la zona urbana (E3, E4, E5), donde la calidad del agua fue media (excepto E3, donde se registra disminución de actividad antropogénica). Lo anterior, se encuentra acorde con los resultados de análisis de clúster por agrupación de estaciones (Figura 2b), donde el primer grupo se relaciona a estaciones ubicadas en zonas con baja densidad poblacional (E1, E2, E6) y con características de buena calidad del agua, para los diferentes periodos climáticos. El segundo grupo, se asocia a estaciones dentro del perímetro urbano (E3, E4, E5), con registro de cambios de calidad buena a media entre el periodo seco y húmedo. Cabe resaltar, el comportamiento de la calidad del agua en la estación E6, a pesar de estar influenciada por el vertimiento de aguas residuales de la laguna de estabilización del municipio, que presenta calidad buena, independiente del periodo de muestreo, indicando que los procesos de autodepuración ambiental aún son eficientes. 
Además, la disminución en la calidad del agua de las estaciones E4 y E5 está relacionada con actividades antrópicas, como explotación de arena en la ribera, vertimiento de aguas residuales y excretas provenientes de la ganaderías porcinas, ubicadas en el perímetro de la ronda hídrica, las cuales, en su gran mayoría, no disponen de sistemas de alcantarillado (PDM, 2020). Fontalvo Julio \& TamarisTurizo (2018) reportaron que los cambios presentados de calidad de agua en sistemas lóticos están influenciados por la variabilidad climática mas no por las actividades antrópicas desarrolladas por los centros poblados, asociado a la capacidad de autodepuración de las fuentes, lo cual, se refleja en la zona de estudio, al presentar, para la mayoría de las estaciones, características de calidad buena, independiente de las diferentes fuentes antrópicas.

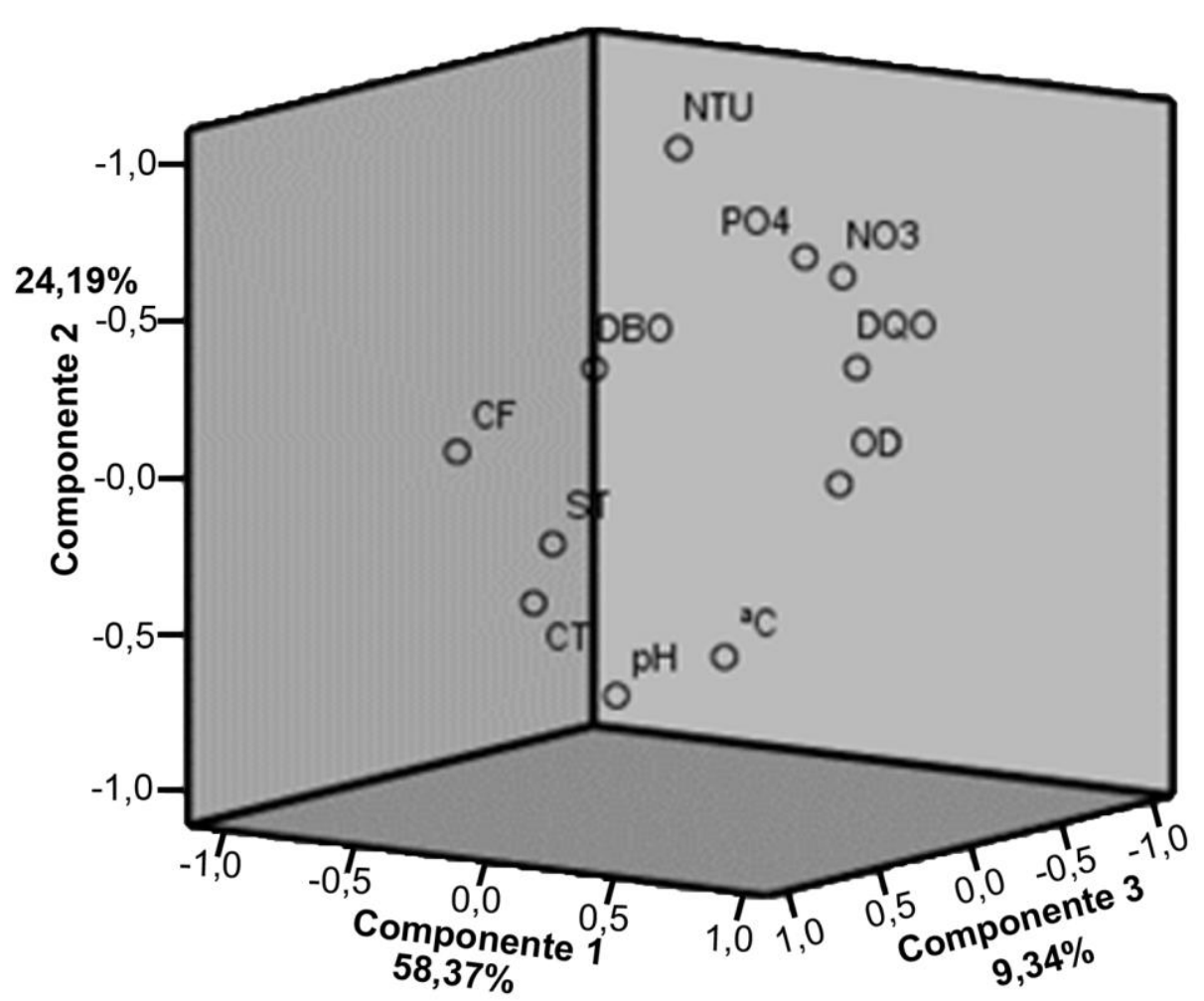

(a)

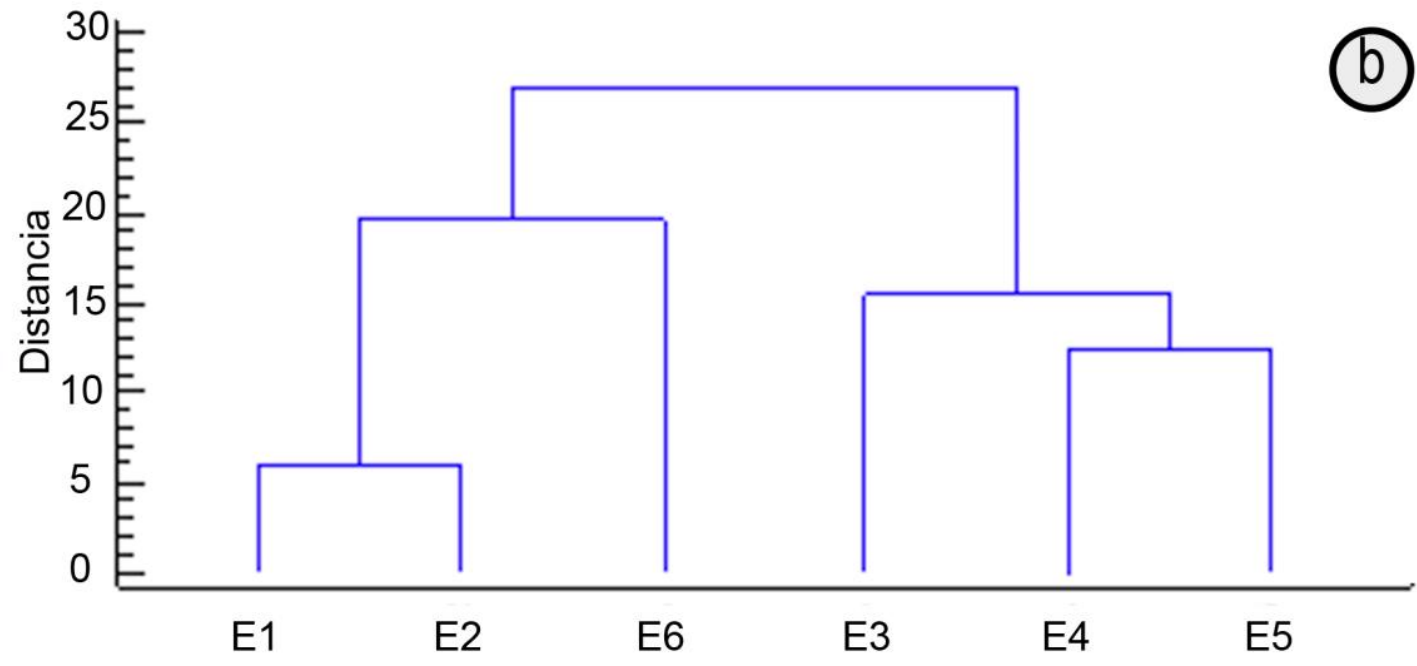

Figura 2. Métodos de agrupamiento: a. Análisis de componentes principales de los parámetros; b. Dendograma estaciones de muestreo (Método de promedio de grupo, euclidiana cuadrada). 


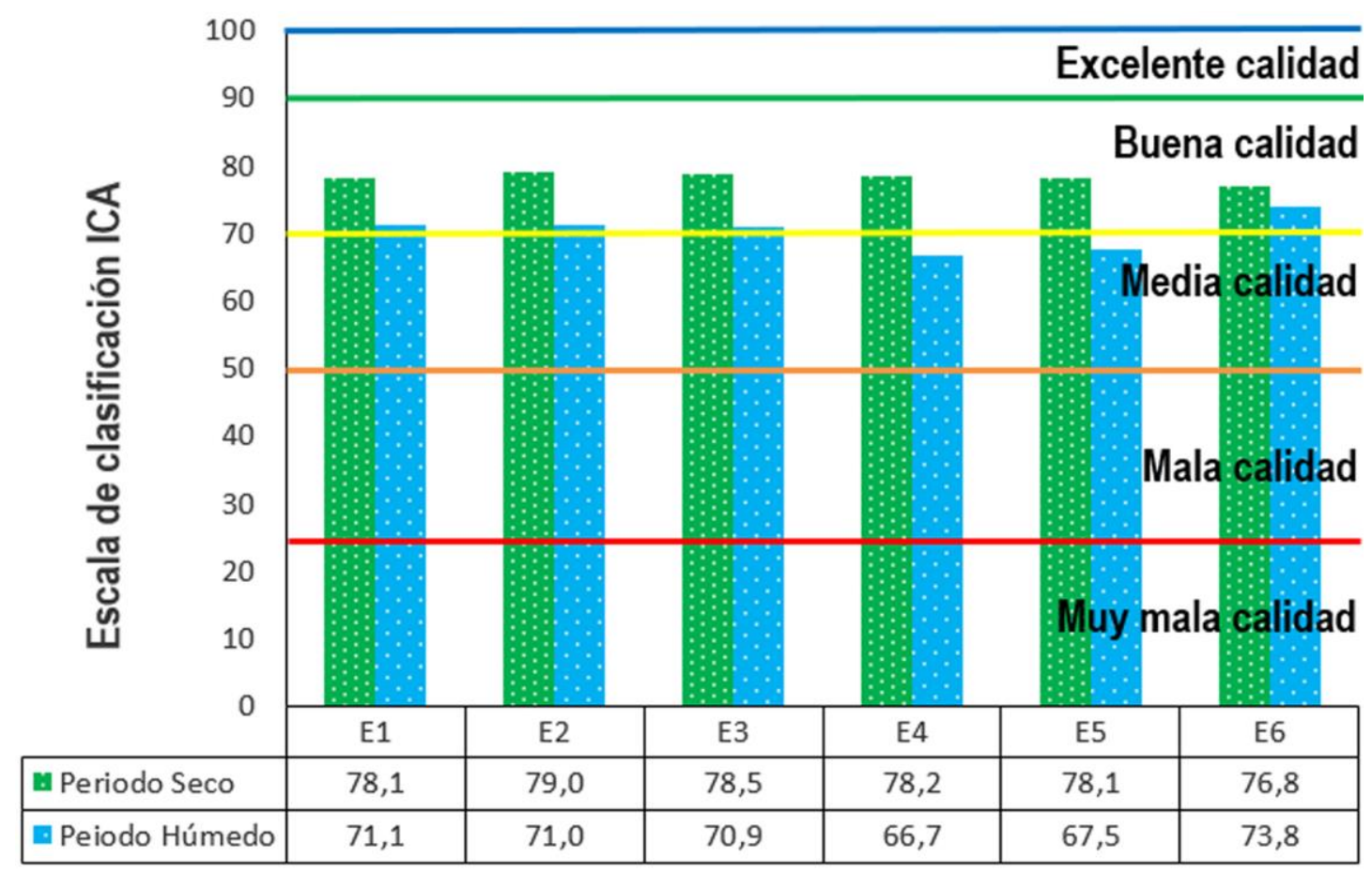

Figura 3. Resultados del ICA en función de las estaciones y periodos de muestreo en la cuenca baja de la quebrada Jui.

Los niveles de clasificación de la calidad del agua fueron similares a los reportes, en el río Otún, en el departamento de Risaralda (Usma Rios et al. 2008); la quebrada La Ayurá, en Antioquia (González Meléndez et al. 2013); cuenca baja del río Córdoba, en Magdalena (Fontalvo Julio \& Tamaris-Turizo, 2018) y quebrada Mamarramos, en Boyacá (Cerón-Vivas et al. 2019). Además, fueron superiores a los reportados en la presa la Boquilla, en Chihuahua-México (Rubio Arias et al. 2014) y cuenca del Lago Taihu-China (Wu et al. 2018). Lo anterior confirma, que el ICA es una herramienta para calcular el potencial de contaminación y clasificar la calidad del agua de manera comprensiva, evidenciando que los cuerpos de agua se encuentran bajo algún grado de contaminación, por materia orgánica, nutrientes y microorganismos (Gil-Marín et al. 2018; Hernández Vargas et al. 2020).

El análisis de componentes principales (ACP) conduce a una reducción de la dimensión inicial del conjunto de datos a tres componentes, que explican el 92,5\% de la variación total (Figura 2a). El primer componente principal (CP1) explicó 58,37\% de la variabilidad total de los datos; las variables de mayor aporte fueron $\mathrm{NO}_{3}$, OD y DQO, relacionado con una fuente antropogénica común, en este caso, aguas residuales domésticas, cuyos contenidos orgánicos e inorgánicos reflejan la estrecha relación de las variables del $\mathrm{CP} 1$ con el comportamiento químico del agua en procesos de oxidación por nutrientes; no obstante, al presentar correlaciones significativas con $\mathrm{PO}_{4}{ }^{3-}, \mathrm{CT}$ y $\mathrm{CF}$ (Tabla 2), se asocia a una fuente antropogénica mixta: escorrentía agrícola, aguas residuales domésticas y excretas del ganado porcino, provenientes del uso de fertilizantes en cultivos, descargas domésticas y materia fecal del ganado porcino. La correlación altamente significativa entre DQO y $\mathrm{NO}_{3}$, revela que la fuente antropogénica de mayor incidencia son las aguas residuales domésticas, asociados a los centros poblados que realizan vertimientos directos a la fuente hídrica. El segundo componente principal (CP2) está relacionado con $\mathrm{PO}_{4}^{3-}$ y Turb, representando el 24,19\% de la variación total; se explica como una fuente antropogénica mixta: escorrentía agrícola y erosión. El uso de fertilizantes fosfatados en los cultivos y la deforestación de la ribera influyen en la presencia de $\mathrm{PO}_{4}^{-3}$ y Turb; sin embargo, la no correlación de la Turb con las diferentes variables evaluadas (Tabla 2), sugiere que la fuente de mayor aporte es la erosión, asociada a la minería de arena y deforestación. El tercer componente principal (CP3) explicó el 9,94\% y está relacionado con ST y DBO5. El CP3 deriva de una fuente antropogénica común, como aguas residuales domésticas. Los CT y CF presentan comportamiento atípico; las cargas moderadas en cada uno de los componentes sugieren la mezcla de diferentes fuentes antropogénicas.

Este estudio permite concluir, que la quebrada Jui presenta ICA con valores de calidad buena, asociados a las bajas concentraciones de parámetros evaluados y la alta capacidad de autodepuración; sin embargo, el análisis multivariado evidenció que las aguas residuales domésticas, escorrentía agrícola, excretas del ganado porcino y minería de arena, son las principales amenazas, que generan efectos negativos en la calidad de sus aguas, en función del tiempo, razón por lo cual, se recomienda implementar programas de monitoreo a diferentes niveles cronológicos y geográficos, así como implementar estudios en la matriz sedimentos, peces, suelos y cultivos, para conocer si las actividades agrícolas desarrolladas en el pasado con la aplicación de plaguicidas prohibidos, actualmente, persisten o se han presentado procesos de bioacumulación y biomagnificación, en función del tiempo, como lo muestra el estudio realizado por Racero Casarrubia et al. (2021), en área de influencia a la zona de estudio. 
Tabla 2. Matriz de correlación de Pearson para parámetros fisicoquímicos y microbiológicos en la microcuenca de la quebrada Jui.

\begin{tabular}{|c|c|c|c|c|c|c|c|c|c|c|c|}
\hline & $\mathrm{T}$ & CF & CT & DBO & DQO & $\mathrm{NO}_{3}{ }^{-}$ & TURB & OD & $\mathrm{pH}$ & $\mathrm{PO}_{4}{ }^{3-}$ & ST \\
\hline $\mathrm{T}$ & 1 & $-0,67$ & $-0,30$ & 0,50 & 0,46 & 0,18 & $-0,64$ & 0,65 & 0,03 & 0,06 & 0,74 \\
\hline $\mathrm{CF}$ & & 1 & 0,77 & $-0,44$ & $-0,92^{*}$ & $-0,78$ & 0,06 & $-0,89 *$ & 0,52 & $-0,56$ & $-0,34$ \\
\hline CT & & & 1 & $-0,61$ & $-0,82^{*}$ & $-0,87^{*}$ & $-0,37$ & $-0,55$ & 0,72 & $-0,78$ & $-0,24$ \\
\hline DBO & & & & 1 & 0,60 & 0,58 & 0,25 & 0,46 & $-0,61$ & 0,56 & 0,78 \\
\hline DQO & & & & & 1 & $0,95^{* *}$ & 0,30 & $0,90 *$ & $-0,81$ & 0,68 & 0,38 \\
\hline $\mathrm{NO}_{3}^{-}$ & & & & & & 1 & 0,57 & 0,72 & $-0,91 *$ & $0,81^{*}$ & 0,20 \\
\hline TURB & & & & & & & 1 & $-0,02$ & $-0,75$ & 0,57 & $-0,25$ \\
\hline OD & & & & & & & & 1 & $-0,60$ & 0,32 & 0,53 \\
\hline $\mathrm{pH}$ & & & & & & & & & 1 & $-0,65$ & $-0,27$ \\
\hline $\mathrm{PO}_{4}{ }^{3-}$ & & & & & & & & & & 1 & $-0,02$ \\
\hline ST & & & & & & & & & & & 1 \\
\hline
\end{tabular}

*correlación significativa con nivel de probabilidad 0,05 .

** correlación altamente significativa con nivel de probabilidad 0,01 .

Conflicto de intereses: El manuscrito fue preparado y revisado con la participación de todos los autores, quienes declaramos no existe conflicto de intereses que ponga en riesgo la validez de los resultados presentados. La financiación de la investigación fue de recursos propios de los investigadores.

\section{REFERENCIAS}

1. AMERICAN PUBLIC HEALTH ASSOCIATION, APHA. 2017. Standards Methods for examination of water and wastewater, 23rd edition. USA. 956p.

2. ANTONIO PAIBA, A.O.; MONROY ÁVILA, E.F. 2015. Modelación hidrodinámica y determinación de la calidad del agua en el río Botello, Facatativá, Cundinamarca, Colombia. Rev. Investig. Agrar. Ambient. 6(1):169-183.

3. CAVANAGH, J.E.; BURNS, K.A.; BURNSKILL, G.J.; COVENTRY, R.J. 1999. Organochlorine pesticide residues in soils and sediments of the Herbert and Burdekin River regions. North Queensland-implications for contamination of the Great Barrier Reef. Marine Pollution Bulletin. 39:367-375.

https://doi.org/10.1016/S0025-326X(99)00058-2

4. CERÓN-VIVAS, A.; GAMARRA, Y.; VILLAMIZAR, M.; RESTREPO, R.; ARENAS, R. 2019. Calidad de agua de la quebrada Mamarramos. Santuario de Flora y Fauna Iguaque, Colombia. Tecnología y Ciencias del Agua (México). 10(6):90-116.

https://doi.org/10.24850/j-tyca-2019-06-04
5. CORPORACIÓN AUTÓNOMA REGIONAL DE LOS VALLES DEL SINÚ Y SAN JORGE, CVS. 2006. Plan de ordenación forestal-cerro Murrucucu, cuenca de la Quebrada Jui: Caracterización física. Ed. Universidad Nacional, sede Medellín (Colombia). 120p.

6. FERIA, J.J.; MARRUGO, J.L.; GONZÁLEZ, H. 2010. Heavy metals in Sinú River, department of Córdoba, Colombia, South America. Rev. Fac. Ing. Univ. Antioquia. 55:35-44.

7. FONTALVO JULIO, F.A.; TAMARIS-TURIZO, C.E. 2018. Calidad del agua de la parte baja del río Córdoba (Magdalena, Colombia), usando el ICA-NSF. Intropica. 13(2):101-111.

http:/ /dx.doi.org/10.21676/23897864.2510

8. GIL-MARÍN, J.A.; VIZCAINO, C.; MONTAÑO-MATA, N.J. 2018. Evaluación de la calidad del agua superficial utilizando el índice de calidad del agua (ICA). Caso de estudio: Cuenca del Río Guarapiche, Monagas, Venezuela. Anales Científicos (Perú). 79(1):111-119. http://dx.doi.org/10.21704/ac.v79i1.1146

9. GONZÁLEZ-DÁVILA, R.P.; VENTURA-HOULE, R.; DE LA GARZA-REQUENA, F.R.; HEYER-RODRÍGUEZ, L. 2019. Caracterización fisicoquímica del agua de la laguna La Vega Escondida, Tampico, Tamaulipas-México. Tecnología y Ciencias del Agua. 10(1):1-28. https://doi.org/10.24850/j-tyca-2019-01-01

10. GONZÁLEZ MELÉNDEZ, V.; CAICEDO QUINTERO, O.; AGUIRRE RAMIREZ, N. 2013. Aplicación de los 
índices de calidad de agua NSF, DINIUS y BMWP en la quebrada la Ayurá, Antioquia, Colombia. Gestión y Ambiente. 16(1):97-108.

11. GUALDRÓN DURÁN, L.E. 2016. Evaluación de la calidad de agua de ríos de Colombia usando parámetros fisicoquímicos y biológicos. Dinámica Ambiental. 1:83-102.

https://doi.org/10.18041/2590-6704/ambiental. 1.2016 .4593

12. HERNÁNDEZ VARGAS, O.; MANCILLA VILLA, O.R.; PALOMERA GARCÍA, C.; OLGUÍN LÓPEZ, J.L.; FLÓRES MAGDALENO, H.; CAN CHULIM, A.; ORTEGA ESCOBAR, H.M.; SÁNCHEZ BERNAL, E.I. 2020. Evaluación de la calidad del agua y de la ribera en dos cuencas tributarias del río Tuxcacuesco, Jalisco, México. Rev. Int. Contam. Ambie. 36(3):689-701. https://doi.org/10.20937/RICA.53595

13. HOYOS CHAVERRA, M.A.; RODRÍGUEZ BARRIENTOS, D.A.; TORRES QUINTERO, J.E. 2018. Evaluación del recurso hídrico superficial en la subcuenca hidrográfica del río frío en el departamento de Cundinamarca: oferta, demanda y calidad del agua. Rev. Investig. Agrar. Ambient. (Colombia). 9(1):128-136.

https://doi.org/10.22490/21456453.2075

14. INSTITUTO DE HIDROLOGÍA, METEOROLOGÍA Y ESTUDIOS AMBIENTALES, IDEAM. 2018. Reporte de avance del Estudio Nacional del Agua. Disponible desde Internet en:

http://www.andi.com.co/Uploads/Cartilla_ENA_\%20 2018.pdf (con acceso el 23/06/2020).

15. LANS, E.; MARRUGO, J.L.; DÍAZ, B. 2008. Estudio de la contaminación por pesticidas organoclorados en aguas de la ciénaga grande del valle bajo del río Sinú. Temas Agrarios. 13(1):49-56.

16. LEWIS, S.E.; SILBURN, D.M.; KOOKANA, R.S.; SHAW, M. 2016. Pesticide behavior, fate, and effects in the tropics: an overview of the current state of knowledge. J. Agric. Food Chem. 64(20):3917-3924.

https://doi.org/10.1021/acs.jafc.6b01320

17. MARRUGO-NEGRETE, J.; PINEDO-HERNÁNDEZ, J.; DÍEZ, S. 2017. Assessment of heavy metal pollution, spatial distribution and origin in agricultural soils along the Sinu River Basin, Colombia. Environmental Research. 154:380-388.

http://dx.doi.org/10.1016/j.envres.2017.01.021

18. MARRUGO-NEGRETE, J.L.; NAVARRO-FRÓMETA, A.E.; URANGO-CARDENAS, I.D. 2014. Organochlorine Pesticides in Soils from the Middle and Lower Sinú River
Basin (Córdoba, Colombia). Air Soil Pollut. 225.No.2053. https://doi.org/10.1007/s11270-014-2053-3

19. MARTÍNEZ SANTOS, P. 2018. Hidrogeología: principios y aplicaciones. Ed. McGraw-Hill (España). 362p.

20. MINISTERIO DE AGRICULTURA. 1974. Resolución 447 de 1974. Disponible desde Internet en:

https://www.ica.gov.co/getdoc/b2e5ff99-bd80-45e8-aa7ae55f0b5b42dc/plaguicidas-prohibidos.aspx (con acceso el 23/11/2020).

21. MINISTERIO DE AMBIENTE, VIVIENDA Y DESARROLLO TERRITORIAL, MAVDT. 2004. Plan nacional de manejo de aguas residuales municipales en Colombia. Disponible desde Internet en:

www.minvivienda.gov.co/ViceministerioAgua/plan (con acceso el 13/06/2020).

22. MINISTERIO DE AMBIENTE, VIVIENDA Y DESARROLLO TERRITORIAL, MAVDT. 2010. Política nacional para la gestión integral del recurso hídrico. Disponible desde Internet en:

http://www.minambiente.gov.co/libro_pol_nal_rec_ hidrico (con acceso el 13/06/2020).

23. MINISTERIO DE AMBIENTE Y DESARROLLO SOSTENIBLE, MADS. 2015. Decreto Único Reglamentario del Sector Ambiente y Desarrollo Sostenible. Decreto 1076 de 2015. Disponible desde Internet en: http://www.corponor.gov.co/NORMATIVIDAD/ decreto1076 (con acceso el 26/06/2020).

24. MINISTERIO DE LA PROTECCIÓN SOCIAL; MINISTERIO DE AMBIENTE, VIVIENDA Y DESARROLLO TERRITORIAL, MPS-MAVDT. 2007. Características, instrumentos básicos y frecuencias del sistema de control y vigilancia para la calidad del agua para consumo humano. Resolución 2115 de 2007. Disponible desde Internet en:

http://www.minambiente.gov.co/pdf/Legislación_del_ agua (con acceso el 26/06/2020).

25. MORA, J.; CALVO, G. 2010. Estado actual de contaminación con coliformes fecales de los cuerpos de agua de la Península de Osa. Tecnología en Marcha (Costa Rica). 23(5):34-40.

26. MORALES-DURÁN, N.; DE LA TORRE-GONZÁLEZ, A.; GARCÍA-SÁNCHEZ, V.; CHÁVEZ, C. 2018. Estudio de la calidad bacteriológica y parámetros fisicoquímicos del agua del Distrito de Riego 023. Tecnología y Ciencias del Agua (México). 9(1):53-67.

https://doi.org/10.24850/j-tyca-2018-01-04 
27. ORTIZ-ROMERO, L.T.; DELGADO-TASCÓN, J.G.; PARDO-RODRÍGUEZ, D.A.; MURILLO-PEREA, E.; GUIO DUQUE, A.J. 2015. Determinación de metales pesados e índices de calidad en aguas y sedimentos del río Magdalena - tramo Tolima, Colombia. Tumbaga. 2(10):4360.

28. PLAN DE DESARROLLO MUNICIPAL, PDM. 2020. Plan de Desarrollo 2020 - 2023. Paz, Desarrollo y Buen Gobierno del Municipio de Tierralta, Departamento de Córdoba. Disponible desde Internet en:

http: / / tierraltacordoba.gov.co/Transparencia/ Plan\%20de\%20Desarrollo\%2020202023/PLAN\%20 DE \%20DESARROLLO \%20MUNICIPAL \% 20 (TIERRALTA)\%202020\%20-\%202023.pdf (con acceso el 10/08/2020).

29. PRADANA PÉREZ, J.A.; GARCÍA AVILÉZ, J.; GALLEGO PICÓ, A.; BRAVO YAGÜE, J.C.; GARCÍA MAYOR, M.A.; PANIAGUA GONZÁLEZ, G. 2019. Criterios de calidad y gestión del agua potable. Ed. UNED, U. Nacional de Educación a Distancia (España). 480p.

30. RACERO CASARRUBIA, J.A.; BALLESTEROS CORREA, J.; MARRUGO-NEGRETE, J.; PINEDO-HERNÁNDEZ, J.J. 2021. Plaguicidas organoclorados en murciélagos (Chiroptera) asociados al bosque húmedo tropical en Córdoba, Colombia. Caldasia. 43(2): Versión de impresión. https://dx.doi.org/10.15446/caldasia.v43n2.84862

31. RESTREPO MEJÍA, A.P.; TOBÓN MEJÍA, O.L. 2002. Manual de calidad de aguas. Escuela de Ingeniería de Antioquia: Facultad de Ingeniería Ambiental. Medellín, Colombia.

32. RICE, C.P.; NOCHETTO, C.B.; ZARA, P. 2002. Volatilization of trifluralin, atrazine, metolachlor, chlorpyrifos, a-endosulfan and B-endosulfan from freshly tilled soil. Journal of Agricultural and Food Chemistry. 50(14):40094017.

https://doi.org/10.1021/jf011571t

33. RUBIO ARIAS, H.O.; ORTÍZZ DELGADO, R.C.; QUINTANA MARTÍNEZ, R.M.; SAUCEDO TERÁN, R.A.; OCHOA RIVERO, J.M.; REY BURCIAGA, N.I. 2014. Índice de calidad de agua (ICA) en la presa la Boquilla en Chihuahua, México. Ecosistemas y Recursos Agropecuarios. 1(2):139150.

34. SHARMA, A.; SHUKLA, A.; ATTRI, K.; KUMAR, M.; KUMAR, P.; SUTTEE, A.; SINGH, G.; BARNWAL, R.P.; SINGLA, N. 2020. Global trends in pesticides: A looming threat and viable alternatives. Ecotoxicology and Environmental Safety (United States). 201:110-812. https://doi.org/10.1016/j.ecoenv.2020.110812
35. SISTEMA DE SELECCIÓN DE BENEFICIARIOS PARA PROGRAMAS SOCIALES, SISBEN. 2009. Censo Municipio de Tierralta, Córdoba.

36. SONI, H.B.; THOMAS, S. 2014. Assessment of surface water quality in relation to water quality index of tropical lentic environment, Central Gujarat, India. Internal J. Environment. 3(1):168-176.

37. SUTADIAN, A.D.; MUTTIL, N.; YILMAZ. A.G.; PERERA, B.J.C. 2016. Development of river water quality indices - a review. Environmental Monitoring and Assessment (Netherlands). 188(58):1-33. https://doi.org/10.1007/s10661-015-5050-0

38. SVINGEN, T.; CHRISTIANSEN, S.; TAXVIG, C.; VINGGAARD, A.M. 2018. Pesticides. En: Skinner, M.K. (ed). Encyclopedia of Reproduction. Ed. Academic Press (United States). p.624-628.

39. SWEETMAN, A.J.; VALLE, M.D.; PREVEDOUROS, K.; JONES, K.C. 2005. The role of soil organic carbon in the global cycling of persistent organic pollutants (POPs): interpreting and modelling field data. Chemosphere. 60(7):959-972.

https://doi.org/10.1016/j.chemosphere.2004.12.074

40. TORRES, P.; CRUZ, C.H.; PATIÑO, P.; ESCOBAR, J.C.; PÉREZ, A. 2010. Aplicación de índices de calidad de agua - ICA orientados al uso de la fuente para consumo humano. Ingeniería e Investigación (Colombia). 30(3):86-95.

41. TOSIC, M.; RESTREPO, J.D.; LONIN, S.; IZQUIERDO, A.; MARTINS, F. 2019. Water and sediment quality in Cartagena Bay, Colombia: Seasonal variability and potential impacts of pollution. Estuarine, Coastal and Shelf Science (Netherlands). 216:187-203.

https://doi.org/10.1016/j.ecss.2017.08.013

42. USMA RIOS, J.M.; VILLEGAS ZULUAGA, C.M.; ARRUBLA VÉLEZ, J.P. 2008. Evaluación del grado de contaminación por pesticidas organoclorados del Río Otún, mediante GCMS. Scientia Et Technica (Colombia). 14(40):234-239.

43. WU, Z.; WANG, X.; CHEN, Y.; CAI, Y.; DENG, J. 2018. Assessing river water quality using water quality index in Lake Taihu Basin, China. Science of The Total Environment (Netherlands). 612:914-922.

https://doi.org/10.1016/j.scitotenv.2017.08.293 